\title{
Ten tips to optimize weaning and extubation success in the critically ill
}

\author{
Boris Jung ${ }^{1,2}$, Rosanna Vaschetto ${ }^{3,4}$ and Samir Jaber ${ }^{2,5^{*}}$ (1)
}

(c) 2020 Springer-Verlag GmbH Germany, part of Springer Nature

Prolonged mechanical ventilation (MV) is associated with complications and prolonged intensive care unit (ICU) stay $[1,2]$. Weaning time (referring to the first attempt to separate the patient from the ventilator, whatever its modality) accounts for up to $50 \%$ of the duration of MV. In this short review, we set out what we consider to be the ten most important tips for accelerating the weaning and extubation process.

\section{Less is more: avoid unnecessary sedation}

One cannot conceive of weaning without optimizing sedation and limiting the use of paralytics. Sedation protocols (nursing-protocolized targeted sedation or daily sedative interruption) have been associated with shorter duration of MV in both medical and surgical patients [3, 4 ] in comparison with no protocols, and are currently recommended by international guidelines [1, 4].

\section{Diaphragm-protective ventilation to prevent respira- tory muscle complications of $M V$}

Prolonged controlled mode ventilation is associated with numerous complications including respiratory muscle dysfunction/atrophy, also called ventilator-induced diaphragm dysfunction [2], and with poor outcome. High tidal volumes, excessive inspiratory efforts and patientventilator asynchronies are associated with both lung and diaphragm injuries. The effort-dependent lung injury has

\footnotetext{
*Correspondence: s-jaber@chu-montpellier.fr

${ }^{5}$ Anesthesiology and Critical Care Department, Saint Eloi Teaching Hospital, Montpellier University Hospital, 80 Avenue Augustin Fliche, 34090 Montpellier, France

Full author information is available at the end of the article
}

been termed "patient self-inflicted lung injury" (PSILI) [5].

\section{Daily screen for spontaneous breathing trial (SBT)}

To be eligible for an SBT under the European guidelines, the condition that led to the patient's intubation must be improving and the patient's vitals must be within physiological ranges with low or no organ support, or within acceptable limits for specific patients [6]. Whether some of these items should be reconsidered remains to be tested in future studies (e.g. should we revise the classical respiratory criteria: $\mathrm{PaO}_{2} / \mathrm{FiO}_{2}>150 \mathrm{mmHg}, \mathrm{FiO}_{2}<40 \%$, PEEP $\left.<8 \mathrm{cmH}_{2} \mathrm{O}\right)$ ?

\section{Which SBT?}

Choosing the best SBT at the bedside is not an easy task and no large study has ever compared different trials considering different clinical vignettes and respiratory physiology patterns. The T-Tube trial is the SBT that closely reflects post-extubation inspiratory effort. However, the goal of the SBT should be, rather, to answer the question "can I wean and extubate my patient with a low risk of reintubation based on this SBT?". When choosing an SBT, it is important to remember that an "easy" trial, characterized by high assistance (e.g. pressure support $7 \mathrm{cmH}_{2} \mathrm{O}$ with PEEP $5 \mathrm{cmH}_{2} 0$ ) and short duration (30 $\mathrm{min})$, is associated with a higher risk of post-extubation respiratory failure than a tougher test $(60-120 \mathrm{~min}$ of T-Tube), which is associated with a higher rate of SBT failure and subsequent delayed extubation [7]. This is the reason why the most recent guidelines suggest that the SBT may be performed if it has a low level of assistance and a short duration (pressure support $7 \mathrm{cmH}_{2} \mathrm{O}$, PEEP $0 \mathrm{cmH}_{2} 0,30 \mathrm{~min}$ ) [1]. 


\section{Protocolized or semi-automated weaning strategy}

Daily and systematic use of a checklist in a ventilator liberation protocol is associated with 25 more ventilatorfree hours and one more ICU-free day compared with no protocol. Whether automated and semi-automated algorithms available on modern ventilators could further increase the ventilator-free days remains uncertain and may be further explored in difficult-to-wean patients. Similarly, proportional modes of ventilation which assist the patient by adapting to his/her effort could be considered in selected patients [8].

\section{Quickly intervene when an SBT fails}

Performing an SBT as soon as the patient becomes "eligible" should be considered as a "stress test", both because it may shorten the duration of invasive MV and because it may unmask one or several undiagnosed conditions (with positive pressure) that should be addressed and treated before the next trial.

\section{Inspiratory muscle load/force generation ratio}

SBT failure reflects an imbalance between inspiratory muscle load and neuromuscular efficiency. Because of specific muscle characteristics and load discrepancies between respiratory and limb muscle groups, the respiratory load/efficiency balance cannot be extrapolated from the examination of limb muscles [9]. In patients in whom the load/efficiency balance is altered, expiratory muscles are recruited, and a better understanding of their function during acute respiratory failure has recently been highlighted [10].

\section{Weaning vs extubation failure risk factors}

Difficult-to-wean patients make up $20 \%$ of the mechanically ventilated critically ill population; being older than 65 years and being affected by cardiopulmonary comorbidities are the main known risk factors [11]. Besides weaning risk factors, Jaber et al. compared the risk of airway-related vs non-airway-related risk factors for reintubation within $48 \mathrm{~h}$ following extubation (defined extubation failure) and reported that three risk factors were specific to airway failure (female sex, duration of ventilation $>7$ days, copious secretions) and two others (non-obese status, SOFA score $\geq 8$ ) to non-airway failure [12].

\section{Post-extubation respiratory support}

Ten to $15 \%$ of patients will need to be reintubated within 48 to $72 \mathrm{~h}$ post extubation [12]. Standard oxygen therapy should probably be used only in easy-to-wean patients with no or few extubation failure risk factors. On the other hand, in high-risk patients, the combination of high-dose non-invasive ventilation (NIV) (at least $12 \mathrm{~h}$ per day for $48 \mathrm{~h}$ following extubation) with high-flow nasal oxygen (HFNO) is associated with less reintubation in comparison with HFNO alone [11]. In low- to moderate-risk patients, prophylactic HFNO has been associated with a lower rate of reintubation than standard oxygen therapy in medical but not surgical patients [13-15]. Likewise, HFNO is probably not inferior to NIV alone in preventing post-extubation respiratory failure [14] and may be considered as a first-line prophylactic respiratory support option in patients with a moderate risk of weaning failure. In expert centers, NIV may also be used as a weaning strategy in patients who failed the SBT, as a way to provide positive pressure without the side effects of the tracheal tube and sedation [16-18].

\section{Tracheostomy}

Early vs late tracheostomy has not been associated with a better prognosis in the general ICU population versus the neurocritically ill population. Patients who may be considered eligible for a late tracheotomy ( $>10$ days of $\mathrm{MV}$ ) are those that maybenefit, for instance, from gradual weaning and constant airway/mucus plugging control.

Weaning and extubation success depends on careful monitoring, especially in high-risk patients, of adequate pain/sedation management, patient/ventilator interaction, and respiratory load/neuromuscular efficiency. A low pressure spontaneous breathing trial of short duration represents the best compromise between unnecessary prolonged MV and hazardous reintubation. Finally, the association between HFNO and NIV may be considered as a post-extubation respiratory support option in selected high-risk patients.

\section{Author details}

${ }^{1}$ Medical Intensive Care Unit, Lapeyronie Teaching Hospital, Montpellier University Hospital, Montpellier, France. ${ }^{2}$ PhyMedExp, INSERM CNRS, Montpellier University, Montpellier, France. ${ }^{3}$ Azienda Ospedaliero Universitaria "Maggiore Della Carità", Anestesia e Terapia Intensiva, Corso Mazzini 18, Novara, Italy.

${ }^{4}$ Università del Piemonte Orientale, Novara, Italy. ${ }^{5}$ Anesthesiology and Critical 
Care Department, Saint Eloi Teaching Hospital, Montpellier University Hospital, 80 Avenue Augustin Fliche, 34090 Montpellier, France.

\section{Compliance with ethical standards}

\section{Conflicts of interest}

SJ reports receiving consulting fees from Drager, Medtronic, Fresenius, Baxter, and Fisher \& Paykel. BJ declares travel fees reimbursement from Hamilton Medical, Bonduz, Switzerland.

\section{Publisher's Note}

Springer Nature remains neutral with regard to jurisdictional claims in published maps and institutional affiliations.

\section{Received: 4 October 2020 Accepted: 13 October 2020}

Published online: 26 October 2020

\section{References}

1. Ouellette DR, Patel S, Girard TD et al (2017) Liberation from mechanical ventilation in critically III adults: an official american college of chest physicians/american thoracic society clinical practice guideline: inspiratory pressure augmentation during spontaneous breathing trials, protocols minimizing sedation, and noninvasive ventilation immediately after extubation. Chest 151:166-180. https://doi.org/10.1016/j.chest.2016.10.036

2. Jaber S, Petrof BJ, Jung B et al (2011) Rapidly progressive diaphragmatic weakness and injury during mechanical ventilation in humans. Am J Respir Crit Care Med 183:364-371. https://doi.org/10.1164/rccm.20100 4-06700C

3. Chanques G, Conseil M, Roger C et al (2017) Immediate interruption of sedation compared with usual sedation care in critically ill postoperative patients (SOS-Ventilation): a randomised, parallel-group clinical trial. Lancet Respir Med 5:795-805. https://doi.org/10.1016/S2213-2600(17)30304 $-1$

4. Devlin JW, Skrobik Y, Gélinas C et al (2018) Clinical practice guidelines for the prevention and management of pain, agitation/sedation, delirium, immobility, and sleep disruption in adult patients in the ICU. Crit Care Med 46:e825-e873. https://doi.org/10.1097/CCM.0000000000003299

5. Yoshida T, Amato MBP, Kavanagh BP (2018) Understanding spontaneous vs. ventilator breaths: impact and monitoring. Intensive Care Med 44:2235-2238. https://doi.org/10.1007/s00134-018-5145-5

6. Boles JM, Bion J, Connors A et al (2007) Weaning from mechanical ventilation. Eur Respir J 29:1033-1056

7. Subirà C, Hernández G, Vázquez A et al (2019) Effect of pressure support vs t-piece ventilation strategies during spontaneous breathing trials on successful extubation among patients receiving mechanical ventilation: a randomized clinical trial. JAMA 321:2175-2182. https://doi.org/10.1001/ jama.2019.7234
8. Jonkman AH, Rauseo M, Carteaux G et al (2020) Proportional modes of ventilation: technology to assist physiology. Intensive Care Med. https:// doi.org/10.1007/s00134-020-06206-Z

9. Jung B, Moury PH, Mahul M et al (2016) Diaphragmatic dysfunction in patients with ICU-acquired weakness and its impact on extubation failure. Intensive Care Med 42:853-861. https://doi.org/10.1007/s0013 4-015-4125-2

10. Shi Z-H, Jonkman A, de Vries $\mathrm{H}$ et al (2019) Expiratory muscle dysfunction in critically ill patients: towards improved understanding. Intensive Care Med 45:1061-1071. https://doi.org/10.1007/s00134-019-05664-4

11. Thille AW, Muller G, Gacouin A et al (2019) Effect of postextubation highflow nasal oxygen with noninvasive ventilation vs high-flow nasal oxygen alone on reintubation among patients at high risk of extubation failure: a randomized clinical trial. JAMA 322:1465-1475. https://doi.org/10.1001/ jama.2019.14901

12. Jaber S, Quintard H, Cinotti R et al (2018) Risk factors and outcomes for airway failure versus non-airway failure in the intensive care unit: a multicenter observational study of 1514 extubation procedures. Crit Care Lond Engl 22:236. https://doi.org/10.1186/s13054-018-2150-6

13. Futier E, Paugam-Burtz C, Godet T et al (2016) Effect of early postextubation high-flow nasal cannula vs conventional oxygen therapy on hypoxaemia in patients after major abdominal surgery: a French multicentre randomised controlled trial (OPERA). Intensive Care Med 42:1888-1898. https://doi.org/10.1007/s00134-016-4594-y

14. Hernández G, Vaquero C, González P et al (2016) Effect of postextubation high-flow nasal cannula vs conventional oxygen therapy on reintubation in low-risk patients: a randomized clinical trial. JAMA 315:1354-1361. https://doi.org/10.1001/jama.2016.2711

15. Leone M, Einav S, Chiumello D et al (2020) Noninvasive respiratory support in the hypoxaemic peri-operative/periprocedural patient: a joint ESA/ESICM guideline. Intensive Care Med 46:697-713. https://doi. org/10.1007/s00134-020-05948-0

16. Vaschetto R, Longhini F, Persona P et al (2019) Early extubation followed by immediate noninvasive ventilation vs. standard extubation in hypoxemic patients: a randomized clinical trial. Intensive Care Med 45:62-71. https://doi.org/10.1007/s00134-018-5478-0

17. Perkins GD, Mistry D, Gates S et al (2018) Effect of protocolized weaning with early extubation to noninvasive ventilation vs invasive weaning on time to liberation from mechanical ventilation among patients with respiratory failure: the breathe randomized clinical trial. JAMA 320:18811888. https://doi.org/10.1001/jama.2018.13763

18. Rochwerg B, Brochard L, Elliott MW et al (2017) Official ERS/ATS clinical practice guidelines: noninvasive ventilation for acute respiratory failure. Eur Respir J. https://doi.org/10.1183/13993003.02426-2016 